\title{
Author Correction: Defects in mtDNA replication challenge nuclear genome stability through nucleotide depletion and provide a unifying mechanism for mouse progerias
}

Riikka H. Hämäläinen (D), Juan C. Landoni (D), Kati J. Ahlqvist, Steffi Goffart, Sanna Ryytty, M. Obaidur Rahman, Virginia Brilhante, Katherine Icay, Sampsa Hautaniemi (D), Liya Wang, Marikki Laiho and Anu Suomalainen (DD

Correction to: Nature Metabolism https://doi.org/10.1038/s42255-019-0120-1, published online 7 October 2019.

In the version of this article initially published, in Fig. 2c,d and Extended Data Fig. 3a,b, the $y$ axes of the dNTP plots were mislabelled. In Fig. $2 \mathrm{c}$,d, the $y$-axis labels read '[dNTP] relative' but should have read '[dNTP] fmol per $\mu$ g mitochondrial protein'. In Extended Data Fig. 3a,b, the $y$-axis units read ' $[\mathrm{dNTP}] \mathrm{uM}$ ' but should have read ' $[\mathrm{dNTP}]$ nmol/million cells'. The errors have been corrected in the HTML and PDF versions of the article.

Published online: 10 August 2020

https://doi.org/10.1038/s42255-020-0255-0

(C) The Author(s), under exclusive licence to Springer Nature Limited 2020 